\title{
Characterizing Health-Related Community Question Answering
}

\author{
Alexander Beloborodov ${ }^{1}$, Artem Kuznetsov ${ }^{1}$, Pavel Braslavski ${ }^{1,2}$ \\ ${ }^{1}$ Ural Federal University, Institute of Mathematics and Computer Science, Russia \\ valan89@gmail.com \\ ${ }^{2}$ Kontur Labs, Yekaterinburg, Russia \\ pbekontur.ru
}

\begin{abstract}
Our ongoing project is aimed at improving information access to narrow-domain collections of questions and answers. This poster demonstrates how out-of-the-box tools and domain dictionaries can be applied to community question answering (CQA) content in health domain. This approach can be used to improve user interfaces and search over CQA data, as well as to evaluate content quality. The study is a first-time use of a sizable dataset from the Russian CQA site Otvety@Mail.Ru.
\end{abstract}

Keywords: community question answering, CQA, consumer health information, content analysis, latent Dirichlet allocation, LDA, Otvety@Mail.Ru

\section{$1 \quad$ Introduction}

According to a 2009 survey, $61 \%$ of American adults look for health information online [2]. A recent study reports that 55\% of Russian adults do not go to the doctor if they are indisposed; in case of self-treatment $32 \%$ seek advice from friends and acquaintances or search information on the Web [4]. Community question answering (CQA) is one of the major destinations for health-related inquiries. Vast amounts of data collected by the CQA sites allow for re-using the "wisdom of crowds" [3].

Our study focuses on questions and answers on health and medicine. This topic is highly exemplary for CQA: search context (e.g. age, gender, or weight of the person the information is sought for) is important; ideally, the answerer has practical experience with the topic; users prefer a personalized answer. The quality of user-generated content (UGC) is essential for answers in the Health category.

Recent studies on health-related CQA data have relied on manual processing of small samples [5], [7]. An approach close to ours is described in [6]: topic modeling is applied to Twitter data in health domain. In our study we use latent Dirichlet allocation (LDA), domain dictionaries, and exploit question-answer structure of the pages to characterize the content. The approach can contribute to a better understanding and representation of CQA data, improved focused search and user interfaces, as well as content quality evaluation on a larger scale. The dataset used in the research comes from a popular Russian CQA site Otvety@Mail.Ru (http://otvet.mail.ru).

adfa, p. 1, 2011.

(C) Springer-Verlag Berlin Heidelberg 2011 


\section{Data}

Otvety@Mail.Ru is a Russian counterpart of Yahoo! Answers (http://answers.yahoo.com/) with similar rules and incentives. The site was launched in 2006 and has accumulated almost 80 million questions and more than 400 million answers by August 2012. ${ }^{1}$ The most remarkable difference from Yahoo! Answers is the two-level directory used at Otvety@ Mail.Ru. The users have to assign their questions to a second-level category using drop-down lists; no hints are provided.

Our data set contains all questions and corresponding answers from the Health and Beauty category from 1 April 2011 to 31 March 2012. The content is quite diverse, covering such subtopics as Tanning, Manicure \& Pedicure, Beauty Salons, Bath \& Massage, Weight Correction, etc. The total number of questions in the dataset is 313,101 . We focus on the largest Diseases and Medicine subcategory that contains $95,002(30.4 \%)$ questions. 133,163 unique users were active in the subcategory during the year (i.e. asked and answered questions). 74,760 (56.1\%) of them have public profiles; age is indicated in $49.6 \%$ of public profiles, and location - in $44.2 \%$ cases (e.g. there are 3,004 users from Moscow region). 50.0\% of public profiles are female, $33.5 \%$ - male, $16.4 \%$ - undefined.

\section{Results}

In this section we briefly describe two approaches we used for data processing: uncovering topics in the collection using LDA and detecting question type based on question-answer content.

We applied GibbsLDA $++^{2}$, an implementation of LDA, to discover topical structure of the collection. (In this case, a document refers to a concatenation of a question and all its answers.) We ran LDA with 100 topics and default parameters $(\alpha=0.5$; $\beta=0.1)$. The most of resulting topics appeared quite meaningful. Out of 100 topics we discarded 29 topics represented by stop-words, digits, and general terms. Table 1 shows some valid topics.

To validate the obtained distributions, we compared dynamics of some topics with infections outbreaks and weather conditions. Figure 2 a shows weekly Acute Respiratory Infection (ARI) rates for Russia from WHO/Europe influenza surveillance ${ }^{3}$ against the share of documents with a high probability of the "flu" topic (the first column in Table 1). Figure $2 b$ juxtaposes the weekly share of "runny nose" threads (the second column in Table 1) started by Moscow inhabitants vs. rainy days count in Russia's capital ${ }^{4}$. The charts demonstrate an acceptable (given the data volume) association between the extracted topics and real-life events.

\footnotetext{
http://otvet.mail.ru/news/\#hbd2012

http://gibbslda.sourceforge.net/

http://euroflu.org/

http://www.gismeteo.ru/
} 
Table 1. Selected topics produced by LDA (top10 terms, originally in Russian)

\begin{tabular}{|c|c|c|c|c|c|}
\hline Fever & nose & cough & hormone & cancer & liver \\
\hline 37 & runny & lung & gland & tumor & gall \\
\hline 5 & drop & bronchitis & endocrinologist & cell & diet \\
\hline 38 & sinusitis & pneumonia & hormonal & stage & bladder \\
\hline flu & ENT & asthma & organism & case & pancreatic \\
\hline $\operatorname{cold} n$ & wash & & thyroid $n$ & dangerous & organ \\
\hline rise & breathe & phlegm & malfunction & oncology & ultrasonic \\
\hline body & snivel & breathe & problem & location & pancreatitis \\
\hline organism & mucosa & syrup & thyroid $a d j$ & mole & acute \\
\hline high & sinus & breath & influence $v$ & even & chronic \\
\hline
\end{tabular}

ARI rate vs. 'flu' topic

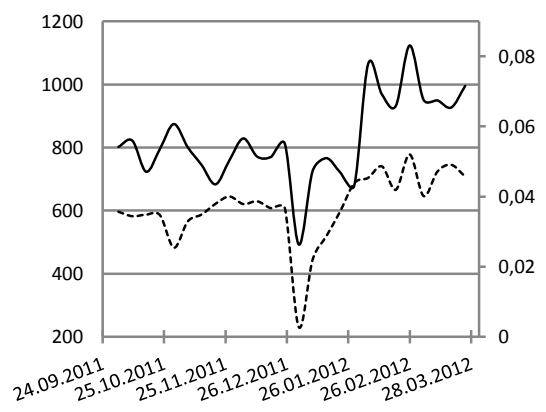

rain vs. 'runny nose' topic

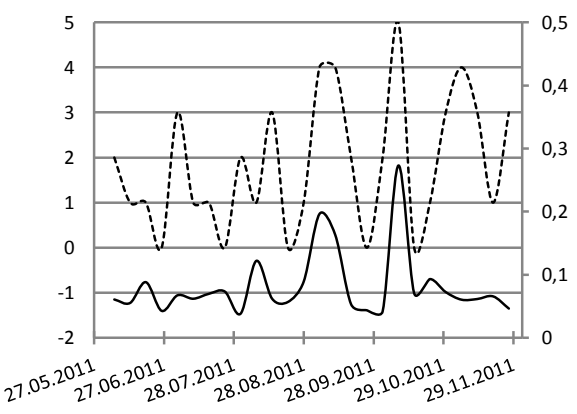

b

Fig. 1. (a) ARI per 100,000 population (dashed line, left axis) vs. 'flu' topic (solid line, right axis); (b) rainy days in Moscow (dashed line, left axis) vs. 'runny nose' questions asked by the users from Moscow region (solid line, right axis)

Question and answer parts of the CQA pages allow us to detect different question types analogous to evidence-directed and hypothesis-directed queries in the Web health search [1]. For example, hypothesis-directed search intent can be associated with a template "disease in question - therapy in answers". To detect these questions we used a list of 1,049 diseases compiled from a reference book for medical assistants and the Russian State Register of Approved Drugs ${ }^{5}$ (11,926 unique trade names effective September 2012). Since complex medicine names and diseases are often misspelled, we implemented a fuzzy search based on character trigrams with a subsequent Levenstein distance check with length-dependent threshold. 15,415 (16.2\%) pages in the dataset contain at least one pair of this kind. Table 2 shows some diseasemedicine pairs along with their frequencies.

5 http://grls.rosminzdrav.ru/ 
Table 2. Sample disease-medicine pairs presented in the Otvety@ Mail.Ru dataset (originally in Russian; asterisks designate drugs with the same active ingredients)

\begin{tabular}{|ll|ll|ll|}
\hline thrush & & angina & & herpes & \\
\hline flucostat $^{*}$ & 155 & iodine & 130 & aciclovir $^{* * *}$ & 307 \\
candid $^{* *}$ & 92 & chamomile & 127 & zovirax & 138 \\
clotrimazole $^{* *}$ & 89 & nitrofural & 111 & wax & 95 \\
fluconazole $^{*}$ & 89 & lugol & 93 & fenistil & 41 \\
diflucan $^{*}$ & 77 & salvia & 70 & valtrex & 34 \\
\hline
\end{tabular}

\section{Conclusions and Future Work}

Our study shows that even a "light" incorporation of domain semantics into CQA analysis can significantly improve understanding of the data. We plan to apply the tested approach to focused health search and representation of the collected data.

We also plan to develop and refine the proposed method. Our plan includes a large-scale quality evaluation of the health-related CQA data. To perform the evaluation, we will use disease classification along with the list of the drugs recommended for each disease. Another direction for future research is to investigate users' followup questions similarly to Web search query sessions.

Acknowledgements. We thank Mail.Ru and Maxim Babich personally for granting us access to the data.

\section{References}

1. Cartright, M.-A., White, R. W., Horvitz, E.: Intentions and Attention in Exploratory Health Search. In: Proceedings of SIGIR'11, pp. 65-74 (2011).

2. Fox, S., Jones, S.: The social life of health information, http://www.pewinternet.org/ /media/ Files/Reports/2009/PIP_Health_2009.pdf

3. Liu, Q., Agichtein, E., Dror, G., Gabrilovich, E., Maarek, Y., Pelleg, D., Szpektor, I.: Predicting Web Searcher Satisfaction with Existing Community-Based Answers. In: Proceedings of SIGIR'11, pp. 415-424 (2011)

4. O Samolechenii i Reklame Lekarstvennykh Preparatov (On Self-Treatment and Drug Advertising), 18.06.2012, http: / fom.ru/obshchestvo/ 10489

5. Oh, S., Worrall, A., Yi, Y. J.: Quality Evaluation of Health Answers in Yahoo! Answers: A Comparison between Experts and Users. In: Proceedings of the American Society for Information Science and Technology, vol. 48(1), pp. 1-3 (2011)

6. Paul, M., Dredze, M.: You Are What You Tweet: Analyzing Twitter for Public Health. In: Proceedings of the Fifth International AAAI Conference on Weblogs and Social Media, pp. 265-272 (2011)

7. Zhang, Y.: Contextualizing Consumer Health Information Searching: an Analysis of Questions in a Social Q\&A Community. In: Proceedings of the $1^{\text {st }}$ ACM International Health Informatics Symposium (IHI '10), pp. 210-219 (2010) 